\title{
Solitary Bone Metastasis Appearing outside the Usual Range of FDG PET/CT in Stage III Lung Cancer: Is Total Body FDG PET/CT Imaging Mandatory?
}

\author{
JC Fanggiday*, W Uyterlinde, MM van den Heuvel and RA Valdés Olmos
}

Department of Heart and Lung, University Medical Center Utrecht, Utrecht, The Netherlands

\begin{abstract}
Nowadays, 18-fluorodeoxyglucose positron emission tomography (18-FDG/PET) and computed tomography (CT) are frequently used in the diagnosis and staging of lung cancer. Usually, FDG-PET/CT images are taken from cranium or skull base to the groin, which covers most of the potential locations of metastasis. We describe three cases of stage IIIB NSCLC with unexpected stage-changing solitary bone metastasis outside the usual range of FDG PET/CT. We discuss whether a total body PET/CT should be performed on a regular basis. In addition, we propose a practical solution that is applicable in each clinic, to assess the whole body "on demand", without compromising the logistics in a busy PET/CT clinic.
\end{abstract}

Keywords: Lung cancer; FDG; PET/CT; Bone metastasis

\section{Introduction}

Lung cancer remains the most common cause of death from cancer in the world with an estimated 1,600,000 new cases and $1,380,000$ deaths in 2008 . The overall 5 -year survival is approximately $15 \%$ [1]. Lung cancer staging determines the treatment strategy. Only $20 \%$ of patients with non-small cell lung carcinoma (NSCLC) qualify for a curative resection [2]. When curative resection of the primary tumor is not possible due to technological reasons, distant metastasis or inoperability due to comorbidities, patients can be treated by radiation therapy, a combination of chemotherapy and radiotherapy or chemotherapy in a palliative setting. 18-Fluorodeoxyglucose positron emission tomography (18-FDG PET) and computed tomography (CT) scans play an important role in staging lung cancer and may serve as a guidance for obtaining material for histopathological diagnosis [3,4]. By combining these, FDG PET/ $\mathrm{CT}$ is nowadays frequently used as a one-stop-shop imaging modality in a fast track setting. Furthermore, FDG PET/CT is used to exclude distant metastasis in patients scheduled to receive chemoradiation.

Usually, FDG PET/CT images are acquired from the base of the skull to the groins, which cover most of the potential locations of metastasis, as they occur most frequently in hilar, mediastinal and/or supraclavicular lymph nodes, bone and the adrenal glands. FDG PET/ $\mathrm{CT}$ is more accurate in detecting bone metastasis in comparison to bone scintigraphy [5], CT [6] and MRI [7]. The other common site of dissemination, the brain, is imaged by MRI, due to the high sensitivity and specificity in comparison to FDG PET/CT [8]. The lower limbs are most often not imaged by FDG PET/CT. We describe three cases of stage IIIB NSCLC where unexpected solitary bone metastasis outside the usual range of FDG PET/CT was found.

\section{Case 1}

A 48 year-old male was referred with a mass in the left lung. FDG PET/CT showed a large metabolic active tumor centrally located in the left upper lobe. Pathological FDG uptake was shown in lymph nodes at level $8,4 \mathrm{~L}$ and $2 \mathrm{R}$ (Figure 1a). Oesophageal ultrasound guided fine needle aspiration showed a NSCLC and stage IIIB disease was confirmed for which concurrent chemoradiation therapy was started consisting of daily dose cisplatin $\left(6 \mathrm{mg} / \mathrm{m}^{2}\right)$ together with radiotherapy (24 times 2.75 Gy [9]). Unfortunately, one month after treatment he developed progressive pain in the left knee. Conventional radiography showed no clear abnormalities (Figure 1b). Because of persistent complaints, MRI was performed, which showed pathologic signal intensity in the left knee, compatible with a bone metastasis (Figure 1c). Palliative chemotherapy was initiated alongside radiation therapy to the left knee.

\section{Case 2}

A 53-year-old female was referred to our hospital with NSCLC of the left lower lobe. FDG PET/CT showed a highly FDG avid tumor with central necrosis in the left lower lobe, as well as FDG avid lymph
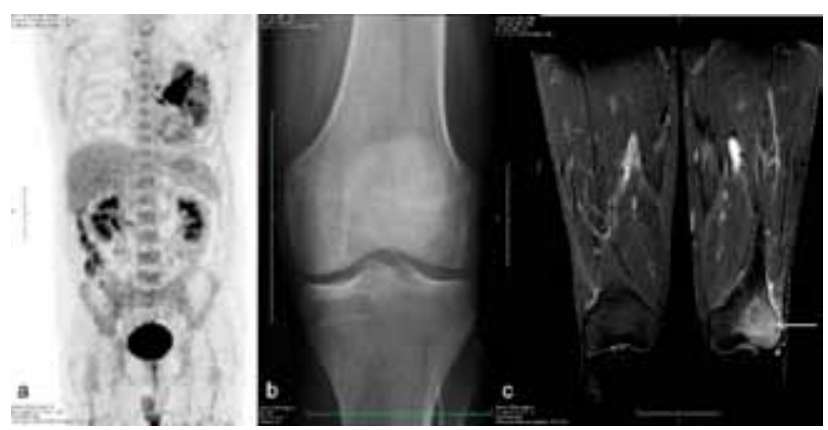

Figure 1: (a) FDG-PET image showing a large metabolic active tumor centrally located in the left upper lobe, with FDG avid lymph nodes at level 8, $4 \mathrm{~L}$ and $2 \mathrm{R}$. (b) No clear abnormalities on left knee X-ray. (c) MRI STIR image showing pathological signal intensity in the lateral condyl of the left femur fitting with bone metastasis.

*Corresponding author: JC Fanggiday, Department of Heart and Lung, University Medical Center Utrecht, Utrecht, The Netherlands, E-mail: jcfanggiday@gmail.com

Received July 14, 2012; Accepted July 26, 2012; Published July 29, 2012

Citation: Fanggiday JC, Uyterlinde W, van den Heuvel MM, Valdés Olmos RA (2012) Solitary Bone Metastasis Appearing outside the Usual Range of FDG PET/ CT in Stage III Lung Cancer: Is Total Body FDG PET/CT Imaging Mandatory? J Nucl Med Radiat Ther 3:135. doi:10.4172/2155-9619.1000135

Copyright: (c) 2012 Fanggiday JC, et al. This is an open-access article distributed under the terms of the Creative Commons Attribution License, which permits unrestricted use, distribution, and reproduction in any medium, provided the original author and source are credited. 
Citation: Fanggiday JC, Uyterlinde W, van den Heuvel MM, Valdés Olmos RA (2012) Solitary Bone Metastasis Appearing outside the Usual Range of FDG PET/CT in Stage III Lung Cancer: Is Total Body FDG PET/CT Imaging Mandatory? J Nucl Med Radiat Ther 3:135. doi:10.4172/21559619.1000135

Page 2 of 3

nodes at level 11L, 10L, 7 and 4R. No distant pathological FDG uptake was shown (Figure 2a). As she was staged as having stage IIIB disease, concurrent chemoradiation was initiated. Because of increased pain in her lower left leg a radiograph was performed. That showed a solitary metastasis in the left fibula head (Figure 2b). Concurrent chemoradiation was terminated. Palliative radiotherapy to the left fibula was started, followed by systemic chemotherapy.

\section{Case 3}

The third patient is a 58 -year-old male, with strong suspicion of lung cancer, who underwent a FDG PET/CT as the initial staging imaging modality. It showed a FDG avid mass in the left upper lobe with involvement of the mediastinum. FDG positive lymph nodes were present at level 10L, 7 and 5 also the right adrenal gland showed elevated FDG uptake (Figure 3a). However, despite repeated biopsies no metastasis was detected in the adrenal gland. It was therefore finally staged as IIIB disease. A radiograph of the right knee was performed three days after start of chemoradiation, because of the onset of pain. A large lytic lesion was seen in the distal part of the right femur. This was

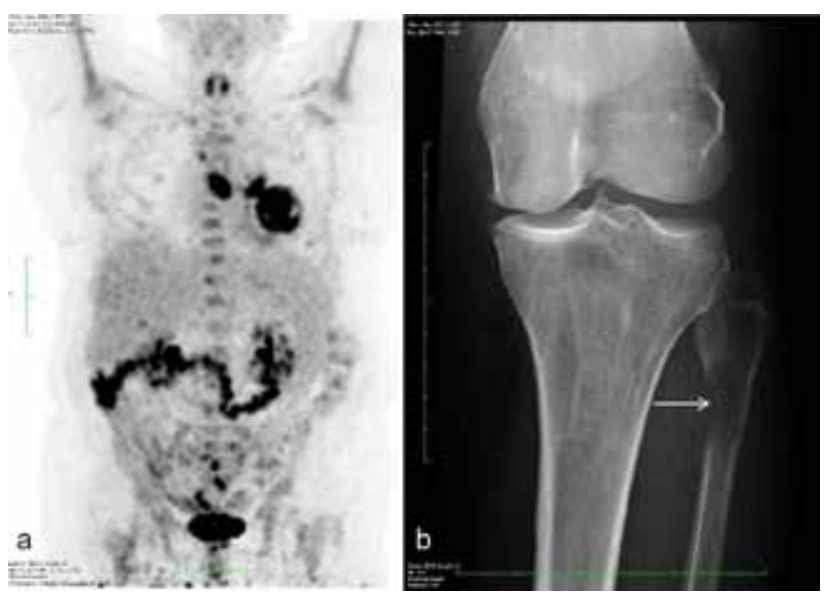

Figure 2: (a) FDG avid lesion in the left lower lobe, with FDG avid ipsilateral, subcarinal and contralateral mediastinal lymph nodes. (b) Lytic bone lesion in the head of the left fibula.
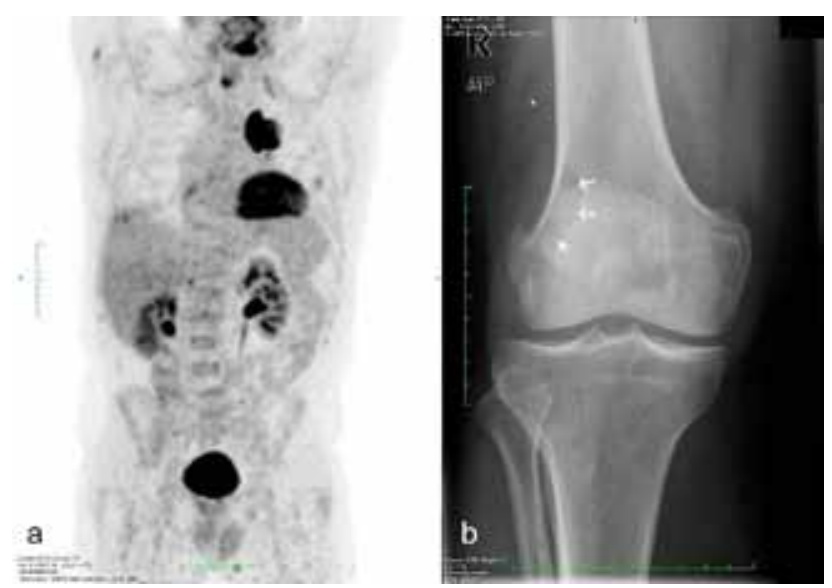

Figure 3: (a) FDG avid mass in the left upper lobe and FDG positive lymph nodes at level 10L, 7, 5. Elevated FDG uptake in the right adrenal gland. (b) Lytic lesion in the distal part of the right femur. consistent with bone metastasis (Figure 3b). Treatment was switched to palliative radiotherapy on the right knee and systemic chemotherapy.

\section{Discussion}

The findings in the patients described in this case series illustrate how solitary metastases, beyond the usual scanning range, impact on the staging and treatment plan of the individual patient. FDG PET/ $\mathrm{CT}$ is increasingly being used for diagnosis and staging of lung cancer, as a "one-stop-shop" imaging modality. This single study, which is performed to characterise the lung tumor itself, provides information about, among others, the mediastinal lymph node status and can exclude adrenal and bone metastasis. FDG PET/CT is more accurate in detecting bone metastasis in comparison to CT, MRI and bone scintigraphy [5-7]. In most hospitals FDG PET/CT is performed from skull base (sometimes from the cranium) to the groin. This raises the question whether or not a total body FDG PET/CT should be used. The described cases arise from a series of 231 consecutive patients with stage III NSCLC who were treated with concurrent chemoradiation over a period of 3 years. The derived incidence is approximately $1 \%$ in patients with this stage of disease.

Every year about 400-500 PET/CT scans are performed in our clinic in order to diagnosis and staging of lung cancer. Performing a total body PET/CT extends the scanning time by ten minutes $(2 \mathrm{~min} /$ bed position and 5 additional bed positions to cover the lower limbs). On a yearly basis this would cost $4000-5000$ minutes. Since a regular $\mathrm{PET} / \mathrm{CT}$ investigation is scheduled with a time span of 30 minutes, performing a total body PET/CT for each patient with suspicion of lung cancer would cost approximately 130-160 PET/CT pitches a year.

In our series solitary stage-changing metastases occurred only once a year; these were later detected using additional imaging. In at least 2 out of the three cases described by us, a careful history of symptoms might have given reasons for suspicion of bone metastases at the time PET/CT was performed. A practical solution to prevent the incorrect staging could be to extend the low dose CT in case of a suggestive history or clinical suspicion of metastasis based on the (vague) pain. With this strategy, it is still possible to assess the whole body "on demand" adequately in a single procedure, without compromising the logistics in a busy FDG PET/CT clinic.

\section{Conclusion}

Solitary stage-changing bone metastases occur outside the usual scope of FDG PET/CT. However, due to the low incidence, performing a total body PET/CT on a regular base would be inefficient. Extending the low dose CT "on demand" may be an option to assess the whole body, without delaying the logistics of a busy FDG PET/CT clinic.

\section{References}

1. Jemal A, Bray F, Center MM, Ferlay J, Ward E, et al. (2011) Global cancer statistics. CA Cancer J Clin 61: 69-90.

2. Groome PA, Bolejack V, Crowley JJ, Kennedy C, Krasnik M, et al. (2007) The IASLC Lung Cancer Staging Project: validation of the proposals for revision of the $\mathrm{T}, \mathrm{N}$, and $\mathrm{M}$ descriptors and consequent stage groupings in the forthcoming (seventh) edition of the TNM classification of malignant tumours. J Thorac Oncol 2: 694-705.

3. Subedi N, Scarsbrook A, Darby M, Korde K, Mc Shane P, et al. (2009) The clinical impact of integrated FDG PET-CT on management decisions in patients with lung cancer. Lung Cancer 64: 301-307.

4. Aukema TS, Valdés Olmos RA, Klomp HM, Teertstra HJ, Belderbos JS, et al. (2009) Evaluation of (18) F-FDG PET-CT for differentiation of pulmonary pathology in an approach of outpatient fast track assessment. J Thorac Oncol 4: $1226-1230$. 
Citation: Fanggiday JC, Uyterlinde W, van den Heuvel MM, Valdés Olmos RA (2012) Solitary Bone Metastasis Appearing outside the Usual Range of FDG PET/CT in Stage III Lung Cancer: Is Total Body FDG PET/CT Imaging Mandatory? J Nucl Med Radiat Ther 3:135. doi:10.4172/21559619.1000135

Page 3 of 3

5. Ak I, Sivrikoz MC, Entok E, Varderelli E (2010) Discordant findings in patients with non-small-cell lung cancer: absolutely normal bone scans versus disseminated bone metastases on positron-emission tomography/computed tomography. Eur J Cardiothorac Surg 37: 792-796.

6. Liu N, Ma L, Zhou W, Pang Q, Hu M, et al. (2010) Bone metastasis in patients with non-small cell lung cancer: the diagnostic role of F-18 FDG PET/CT. Eur J Radiol 74: 231-235.

7. Qu X, Huang X, Yan W, Wu L, Dai K (2012) A meta-analysis of ${ }^{18}$ FDG-PET-CT,
${ }^{18} \mathrm{FDG}-\mathrm{PET}, \mathrm{MRI}$ and bone scintigraphy for diagnosis of bone metastases in patients with lung cancer.Eur J Radiol 81: 1007-1015.

8. Krüger S, Mottaghy FM, Buck AK, Maschke S, Kley H, et al. (2011) Brain metastasis in lung cancer. Comparison of cerebral MRI and 18F-FDG-PET/CT for diagnosis in the initial staging. Nuklearmedizin 50: 101-106.

9. Belderbos J, Uitterhoeve L, van Zandwijk N, Belderbos H, Rodrigus $P$ et al. (2007) Randomised trial of sequential versus concurrent chemo-radiotherapy in patients with inoperable non-small cell lung cancer (EORTC 08972-22973). Eur J Cancer 43: 114-121. 